\title{
THE STRUCTURE OF STABLE PENTABORANE
}

\section{By Kenneth Hedberg, Morton E. Jones and Verner Schomaker \\ Gates and Crellin laboratories of Chemistry, * California Institute of Tech- nology, Pasadena, California}

Communicated by Linus Pauling, June 2, 1952

Stable pentaborane, $\mathrm{B}_{5} \mathrm{H}_{9}$, is one of the boron hydrides, a short, comparatively little studied series of extraordinary compounds for which a satisfactory elementary valence theory is lacking. In 1947 we decided to undertake new electron diffraction studies of the molecular structures. The early diffraction work and most of the theoretical discussion had been too much influenced (it now seems) by unfortunate analogies to ordinary valence compounds, and it had become reasonably clear that at least the old, ethane-like structure for diborane was incorrect and that a bridge structure (I) was more likely. In the case of $\mathrm{B}_{5} \mathrm{H}_{9}$, also, the structure from<smiles></smiles>

(I)

the previous diffraction study ${ }^{1}$ was not in complete agreement with the appearance of the photographs, one of which was available to us. The bridge structure of diborane has now been well established, ${ }^{2}$ the crystal structure of decaborane $\left(\mathrm{B}_{10} \mathrm{H}_{14}\right)$ has been determined, ${ }^{3}$ and the $\mathrm{B}_{5} \mathrm{H}_{9}$ structure has been determined, both from the gas diffraction pattern in the work here to be described ${ }^{4}$ and from an x-ray study of the crystal by Dulmage and Lipscomb. ${ }^{5}$ The most impressive attempt at a theory of the compositions and structures, however-Pitzer's protonated double bond theory, ${ }^{6}$ which based the structures of all the boron hydrides on diborane bridges and on some plausibly assumed conjugation properties of these bridges-has been a casualty: each of the new boron hydride structures has shown little over-all relation to the previous ones and neither involves the diborane bridge.

The Structure Determination.-The method used has been outlined in recent reports from this laboratory. ${ }^{7}$

New photographs were taken with samples kindly provided by Professor H. I. Schlesinger of the University of Chicago and by Doctor I. Shapiro of the Naval Ordnance Test Station, Pasadena. The camera distance was $10.94 \mathrm{~cm}$. and the electron wave-length $0.0608 \AA$. Independent visual interpretations of the photographs were made by two observers (see Fig. 1).

The radial distribution curves, showing only two strong peaks, at $1.74 \AA$. $(\mathrm{B}-\mathrm{B})$ and $2.57 \AA$. (B $\cdots \mathrm{B}$ and $\mathrm{B} \cdots \mathrm{H}$ ), exclude both the structure advocated in the original study ${ }^{1}$ (II) and that proposed by Pitzer $^{6}$ (III): 
<smiles>B1BBBB1</smiles>

(II)<smiles>[BH]B1BBB1</smiles>

(III)

II would requre significant $B \cdots B$ interactions at $1.74 \sqrt{2}=2.46 \AA$. and at $2 \times 1.74 \sin 135^{\circ} / 2=3.22 \AA$., at least if it were normally rigid, and III at an average of $2 \times 1.74 \sin 108^{\circ} / 2=2.82 \AA$. (The original specification of III would also require the $1.74 \AA$. peak to be obviously doubled.) The radial distribution curves did not lead directly to the structure, mainly because neither the relative areas of the widely separated main peaks nor the indicated absence of minor interactions outside them could be relied upon.

Nevertheless, the radial distribution information provided a starting point for a more detailed analysis of the visual curves themselves. This analysis first showed that the observed doubled character of maximum 9-10 requires two groups of $B-B$ interactions, of about equal weight, separated by $0.11 \pm 0.01 \AA$. Even then, the outer part of the observed intensity curve, including max. 9-10, could not be reproduced without severely restricting the distribution of weights and distances within the $2.57 \AA$. radial distribution peak, either by making the distribution essentially continuous (corresponding to severe "temperature" factors) or in other ways which, given the B-B split, were fairly obvious. Finally, when this was done on the assumption that the $2.57 \AA$. peak was due mainly to rigid $B \cdots B$ interactions, it appeared that the $B-H$ terms were probably also split, by about $0.15 \AA$. into two groups of about equal weight. Corresponding to this distance information three unsymmetrical arrangements of the boron atoms, a puckered five-membered ring, a dimethylcyclopropanelike arrangement, and an ethylcyclopropane-like arrangement. all actually rather closely similar, were found.

Before constructing and testing actual models based on these arrangements of boron atoms (the theoretical intensity curves already calculated lacked the $\mathrm{B} \cdots \mathrm{H}$ terms), we decided to re-examine the tetragonal pyramid arrangement, which had been considered but rejected in the original diffraction study, had more recently been further advocated by Pauling, ${ }^{8}$ and, unlike our unsymmetrical arrangements, was in agreement with recent indications of high symmetry from spectroscopic ${ }^{9}$ and calorimetric ${ }^{10}$ data. The $2.57 \AA$. peak now had to be attributed mainly to $\mathrm{B} \cdots \mathrm{H}$ rather than $B \cdots B$ interactions, contrary to our previous assumption, ${ }^{11}$ but with the help of the previous analysis a suitable disposition of hydrogen atoms was readily found (Fig. 2) and all others of full symmetry $\left(\mathrm{C}_{4 v}\right)$ were ten- 


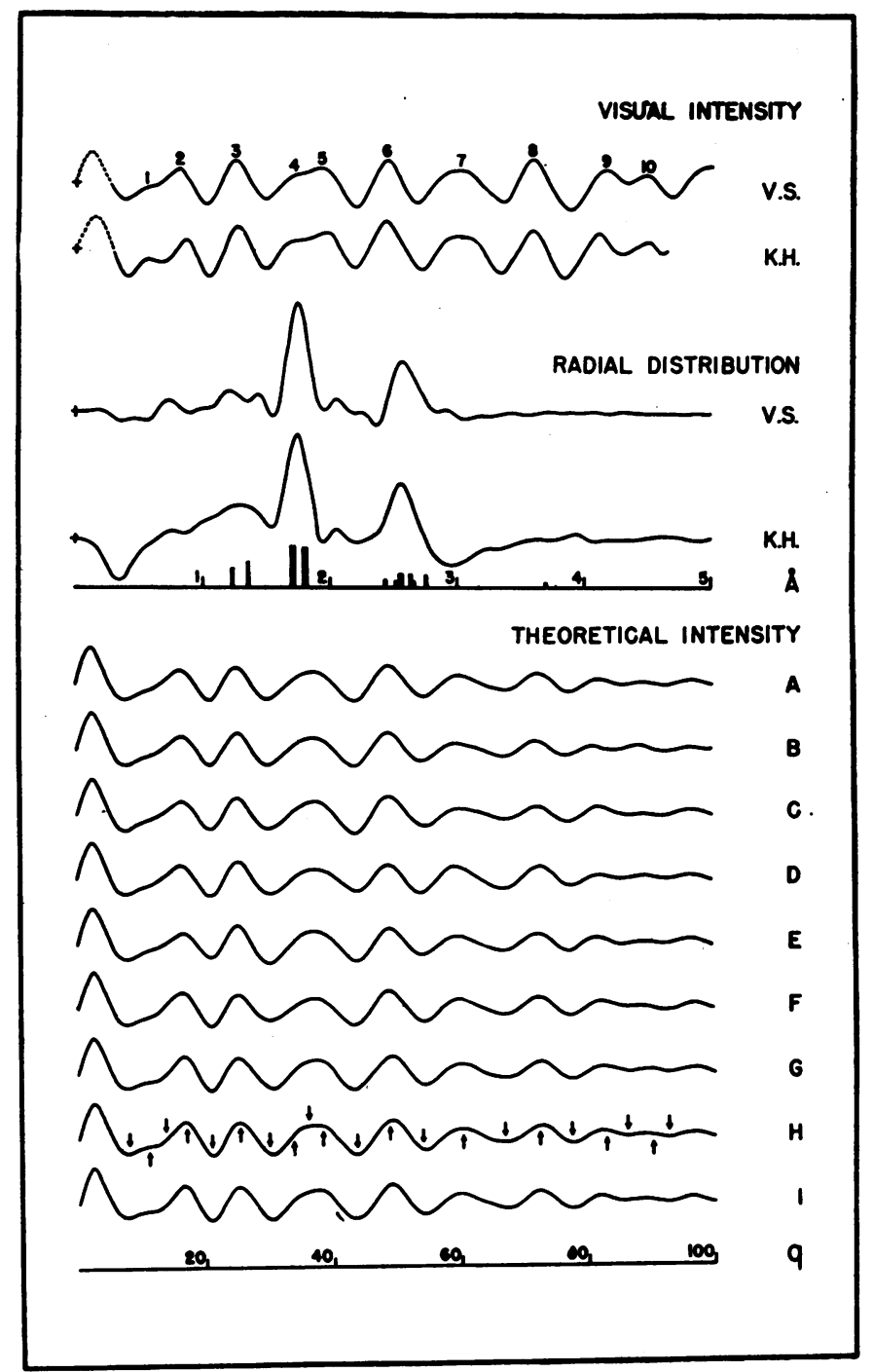

FIGURE 1.

Electron diffraction curves. The theoretical intensity curves are for the following pyramidal models:

$\begin{array}{cccccc}\text { Curves } & \text { B-Hav./B-Bav. } & \text { B-B split } & \text { B-H split } & <\text { B }_{1} \mathbf{B}_{2} \mathrm{~B}_{2} & \begin{array}{c}\text { external dihedra. } \\ \left\langle\mathrm{B}_{1} \mathrm{~B}_{2} \mathrm{~B}_{2}-\mathrm{B}_{2} \mathrm{~B}_{2} \mathrm{H}_{3}\right.\end{array} \\ A & 1.275 / 1.740 & 0.100 & 0.150 & 90^{\circ} & 180^{\circ} \\ B & 1.275 / 1.745 & 0.110 & 0.150 & 90^{\circ} & 180^{\circ} \\ C & 1.275 / 1.740 & 0.100 & 0.050 & 90^{\circ} & 180^{\circ} \\ D & 1.275 / 1.740 & 0.100 & 0.250 & 90^{\circ} & 180^{\circ} \\ E & 1.225 / 1.740 & 0.100 & 0.150 & 90^{\circ} & 180^{\circ} \\ F & 1.325 / 1.740 & 0.100 & 0.150 & 90^{\circ} & 180^{\circ} \\ G & 1.275 / 1.740 & 0.100 & 0.150 & 115^{\circ} & 180^{\circ} \\ H & 1.275 / 1.740 & 0.100 & 0.150 & 115^{\circ} & 190^{\circ} \\ I & 1.275 / 1.740 & 0.100 & 0.150 & 125^{\circ} & 190^{\circ}\end{array}$


tatively eliminated. The new structure, with the $\mathrm{B}_{2}-\mathrm{B}_{2}$ and $\mathrm{B}_{2}-\mathrm{H}_{3}$ distances the longer of their respective kinds, seemed plausible and met with immediate success.

All except $\mathrm{H} \cdots \mathrm{H}$ terms were included for the theoretical intensity curves. The coefficients $a_{i j}$ of the temperature factors $\exp \left(-a_{i j} q^{2}\right)$ were taken as 0.00016 for $\mathrm{B}_{2}-\mathrm{H}_{2}$ and $\mathrm{B}_{1}-\mathrm{H}_{1}, 0.00028$ for $\mathrm{B}_{2}-\mathrm{H}_{3}, 0.00060$ for $\mathrm{B} \cdots \mathrm{H}$, and zero otherwise, as for diborane, ${ }^{2 b}$ and the effective value 1.25 was used for $Z_{H}$. Of the selection of curves shown in Fig. 1, $G, H$, and $I$ are acceptable, $A, B$, and $C$ are doubtful, and $D, E$, and $F$ are unacceptable. Important items for these conclusions are the depth of min. 4 , the shape of doublet $4-5$, the relative intensities of minima $6,7,8$, and 9 , the shape of max. 7$\min .8$, and the position and shape of doublet $9-10$. For the best curves, the only point of substantial disagreement concerns the heights of the first three main maxima; it is almost inconsequential for the parameter determination and probably arises from an underestimate, such as could be expected, of the height of the broad inner max. 1-2.

In terms of $\mathrm{B}-\mathrm{B}_{\mathrm{av}} .=1.740 \AA$. the best shape parameter values and estimated limits of error, together with the ranges for which intensity curves were calculated, are: $\mathrm{B}-\mathrm{H}_{\mathrm{av}}, 1.288 \pm 0.044 \AA$. (1.22-1.35 $\AA$.); $\mathrm{B}-\mathrm{H}_{\mathrm{split}}, 0.125 \pm 0.090 \AA$. $\left(0.05-0.35 \AA\right.$.) $; \mathrm{B}-\mathrm{B}_{\mathrm{split}}, 0.105 \pm 0.010 \AA$. (0.09-0.12 $\AA$.) ; $\angle \mathrm{B}_{1}-\mathrm{B}_{2}-\mathrm{H}_{2}, 120 \pm 20^{\circ}\left(85-125^{\circ}\right)$; and external dihedral angle $\mathrm{B}_{1} \mathrm{~B}_{2} \mathrm{~B}_{2}-\mathrm{B}_{2} \mathrm{~B}_{2} \mathrm{H}_{3}, 187 \pm 10^{\circ}\left(165-200^{\circ}\right)$, all for the assumed $C_{4 v}$ symmetry. These values and the values of $\left\langle q_{\text {oalc. }} / q_{\text {obs. }}\right\rangle_{\text {av. }}$ (see table 1 for an example) lead to the following results for the bond lengths: $\mathrm{B}_{1}-\mathrm{B}_{2}, 1.700 \pm 0.017 \AA . ; \mathrm{B}_{2}-\mathrm{B}_{2}, 1.805 \pm 0.014 \AA$. $; \mathrm{B}_{1}-\mathrm{H}_{1}$ and $\mathrm{B}_{2}-\mathrm{H}_{2}$, $1.234 \pm 0.066 \AA$. $\left(\mathrm{B}_{1}-\mathrm{H}_{1}=\mathrm{B}_{2}-\mathrm{H}_{2}\right.$ assumed $)$; and $\mathrm{B}_{2}-\mathrm{H}_{3}, 1.359 \pm 0.077 \AA$.

The limits of error are conservative except that no allowance has been made for the possible effects on the angle determinations of our rough assumption that the previous guess for $a_{B} \ldots H$ in diborane should apply to $\mathrm{B}_{5} \mathrm{H}_{9}$, for all the different $\mathrm{B} \cdots \mathrm{H}$ terms. The concentration of all the $\mathrm{B} \cdot \mathrm{H}$ distances within the $2.57 \AA$. peak makes the question of interaction between temperature factor and distance parameters more serious than usual, but the boron parameters and probably the $\mathrm{B}-\mathrm{H}$ distances should not be much affected, since they are determined largely by the outer part of the pattern, where the $B \cdots H$ contribution is in any case small. It may be noted that the crystal ${ }^{5}$ and gas values for the bond angles and bond lengths in $\mathrm{B}_{5} \mathrm{H}_{9}$ are in good agreement except for the $\mathrm{B}-\mathrm{B}$ lengths, for which the crystal values $(1.66 \pm 0.02 \AA$. and $1.77 \pm 0.02 \AA$.) are shorter than ours by possibly significant amounts compared to the limits of error. Our B-B lengths, however, are in good agreement with the preliminary results $1.69 \AA$. and $1.80 \AA$. of a recent microwave investigation, ${ }^{12} \mathrm{from}$ which none of the other parameter values have yet been reported.

The $\mathrm{x}$-ray confirmation of the structure type, which was communicated to us during our parameter determination, made unnecessary any further study of other possibilities, including the unsymmetrical ones described above. The high over-all symmetry and especially the $C_{4 v}$ skeletal symmetry have also been confirmed by the microwave investigation. ${ }^{12}$

Discussion.-The $\mathrm{B}_{5} \mathrm{H}_{9}$ structure has high ligancies, two for the bridge hydrogen atoms, five for the apical boron atom, and six for the basal boron 
atoms, in agreement with the principle ${ }^{13}$ that electron deficiency gives rise to structures showing ligancies in excess of the respective numbers of suitable atomic orbitals. For the count of ligands, we take the direct $B-B$ interaction of a bridge bond as bonding, although the related $B \cdots B$ interaction in diborane is often regarded as not bonding. We believe our assumption is the more likely one in view of the comparatively short $B-B$ distance. It also makes the ligancies of hydrogen and boron in the boron hydrides and other high-ligancy compounds of boron more uniformly consistent with the high-ligancy principle, and is the natural assumption to make if these compounds are to be related to ordinary covalent compounds in terms of resonance, following Pauling's discussion of the metals. ${ }^{14}$ Pauling's relation $r_{n}=r_{1}-0.300 \log _{10} n$ yields an attractive correlation of the actually very widely varying bond distances, ${ }^{15}$ as well as inferences about certain other aspects of the structures. For example, the boron radius which would be exactly compatible with the bond distances of our preliminary report for $\mathrm{B}_{5} \mathrm{H}_{9}$ is $0.795 \AA$., in excellent agreement with the average $0.794 \AA$. obtained from all these compounds.

The basal boron atoms of $\mathrm{B}_{5} \mathrm{H}_{9}$ and the apical boron atoms of $\mathrm{B}_{10} \mathrm{H}_{14}$ form just the same set of bonds; similarly, the apical boron atoms of $\mathrm{B}_{5} \mathrm{H}_{9}$ resemble the boron atoms of the calcium boride structure in an octahedron arrangement except for replacement of external $B$ by H. As King and Lipscomb pointed out, ${ }^{16}$ moreover, the whole $\mathrm{B}_{5} \mathrm{H}_{9}$ structure is related to the calcium boride

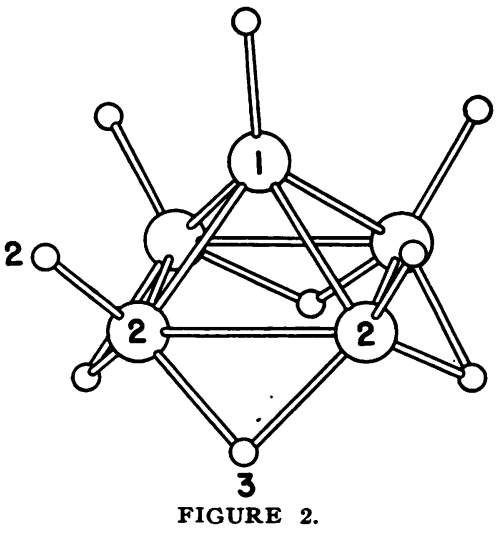

The $\mathrm{B}_{5} \mathrm{H}_{9}$ structure. structure in almost precisely the same way as the $\mathrm{B}_{10} \mathrm{H}_{14}$ structure is related to the boron carbide structure. ${ }^{17}$ We may add that the basal boron atoms of $\mathrm{B}_{5} \mathrm{H}_{9}$ and all the boron atoms of decaborane, as well as the boron atoms of boron carbide and the icosahedron atoms of elementary boron (in the modification of known structure ${ }^{18}$ ), all have six ligands in the icosahedron arrangement, with bond angles approximating the ideal values of $60^{\circ}, 108^{\circ}$, and $121^{3} / 4^{\circ}$ about as well as would seem possible under the constraints imposed by differing bond lengths and incompatible over-all symmetries. ${ }^{19}$ Accordingly, it seems reasonable to suggest that these structures all reflect a strong tendency for sexiligated boron to adopt approximately the ideal icosahedron arrangement.

The occurrence of the icosahedron and octahedron arrangements is remarkable because they are notably anisotropic, in violation of what might be expected to result from the $s p^{2}$ (and $s p^{3}$, for the hydrides) hybrid 
orbitals on which the bonding is presumably mainly based and because there is surely no lack of more conventional alternatives. For elementary boron, for example, ordinary octahedral coordination in the simple cubic structure would seem suitable, especially in view of its frequent occurrence in complex structures for other atoms which are regarded as forming six half-bonds. ${ }^{20}$ To be sure, the icosahedron and octahedron arrangements would seem less anisotropic if the external bonds were stronger than the internal bonds, as indeed is the general indication for $\mathrm{B}_{5} \mathrm{H}_{9}$ and decaborane. For the basal boron atoms of $\mathrm{B}_{5} \mathrm{H}_{9}$, for example, the bridge $\mathrm{B}-\mathrm{H}$, bridge $B-B$, and slant $B-B$ bonds have the respective Pauling bond numbers $0.46,0.45$, and 0.67 , with a total of 2.49 , or only about three times the bond number 0.77 of the $\mathrm{B}-\mathrm{H}$ external bond. ${ }^{15}$ But for boron carbide, ele-

TABLE 1

Comparisons of Observed and Calculated Positions of Maxima and Minima for MODEL $\mathbf{H}$

\begin{tabular}{|c|c|c|c|c|c|c|c|c|}
\hline \multirow{3}{*}{ No. } & & & \multirow{2}{*}{\multicolumn{2}{|c|}{+4}} & \\
\hline & & & & & & & \multicolumn{2}{|c|}{$\overline{M A x}$ K. н. } \\
\hline & MAX. & MIN. & MAX. & MIN. & MAX. & MIN. & MAX. & MIN. \\
\hline 1 & 11.19 & 7.76 & 10.89 & 8.06 & $(0.956)$ & $(0.979)$ & $(0.983)$ & $(0.943)$ \\
\hline 2 & 16.41 & 13.31 & 17.31 & 13.88 & $(1.012)$ & $(0.939)$ & $(0.959)$ & $(0.901)$ \\
\hline 3 & 25.00 & 20.47 & 25.34 & 21.03 & 1.012 & 1.021 & 0.998 & 0.994 \\
\hline 4 & 33.87 & 29.70 & 33.33 & 29.77 & $(1.025)$ & 1.024 & $(1.041)$ & 1.021 \\
\hline 5 & 38.52 & 35.41 & 39.66 & 36.42 & $(0.971)$ & $(1.011)$ & $(0.943)$ & $(0.983)$ \\
\hline 6 & 48.70 & 43.46 & 48.59 & 43.62 & 1.008 & 0.999 & 1.011 & 0.995 \\
\hline 7 & 59.51 & 53.53 & 60.45 & 54.25 & 1.000 & 1.014 & 0.991 & 1.007 \\
\hline 8 & 72.66 & 66.95 & 71.87 & 66.61 & 0.998 & 1.004 & 1.009 & 1.009 \\
\hline 9 & 83.29 & 78.03 & 82.23 & 76.50 & $(0.984)$ & 0.993 & $(0.997)$ & 1.013 \\
\hline 10 & 90.07 & 86.50 & 89.97 & 85.57 & $(0.989)$ & 0.991 & $(0.991)$ & 1.002 \\
\hline \multirow[t]{3}{*}{11} & $\cdots$ & 93.23 & $\cdots$ & 91.89 & $\cdots$ & 0.992 & $\cdots$ & 1.007 \\
\hline & & & \multicolumn{2}{|c|}{ Average, 12 feature } & \multicolumn{2}{|c|}{1.0047} & \multicolumn{2}{|c|}{1.0048} \\
\hline & & & \multicolumn{2}{|c|}{ Average deviation } & \multicolumn{2}{|c|}{0.009} & \multicolumn{2}{|c|}{0.007} \\
\hline
\end{tabular}

mentary boron, and calcium boride there is no definite indication one way or the other. Altogether, a proper understanding of the details of the bonding is lacking.

Nevertheless, it seems unlikely that the immediate bond arrangement in these structures is superior. Instead, the essential point may be that they allow an increase in ligancy without a corresponding increase (or even with a decrease) in the number and severity of close non-bond interactions: compare, for example, the joined icosahedron unit of the boron and boron carbide structures with the simple cubic structure. In the latter, each atom has twelve next-nearest neighbors related to it by $90^{\circ}$ bond angles, whereas the icosahedron atom has only five internal next-nearest neighbors at $108^{\circ}$ and five external next-nearest neighbors at $122^{\circ}$. This strongly suggests that the next-nearest interactions are repulsive and important 
and that the high-ligancy principle should be revised to say that the high ligancies tend to be achieved in such a way as to minimize the numbers and maximize the distances of next-nearest neighbors, even if the resulting bond arrangements would appear by standards of ordinary covalence to be unduly strained. It may also account for the lack of apparent extra strength of the external bonds where two octahedra or two icosahedra are joined: for the icosahedron, again, each external bond would be opposed principally by ten next-nearest interactions at $122^{\circ}$ and ten second-nearest interactions (assuming the staggered orientation of groups about the external bond) of the type

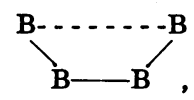

whereas each internal bond is opposed (a full counting shows) by only one internal next-nearest interaction at $108^{\circ}$, two external next-nearest interactions at $122^{\circ}$, and one external interaction of the type

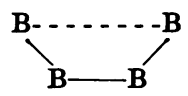

in the opposed orientation. The present situation is evidently related to the cases of cyclopropane and cyclobutane, ${ }^{21}$ where the energy and $\mathrm{C}-\mathrm{C}$ bond length in cyclobutane are both greater than normal, apparently because of cross-ring repulsion, while in cyclopropane, in which the repulsion is avoided by formation of the three-membered ring, the bond length is less than normal and the energy still greater than normal, both apparently in consequence of the angle strain. In the high-ligancy boron compounds the relationships are no doubt different, especially because of the complicated resonance situation; nevertheless, the importance of next-nearest neighbor repulsions seems to be verified and there is the additional indication that angle-strain shortening of the internal bonds may also occur. For the calcium boride structure, of course, the role of the metal atoms has also to be considered.

We should like to express our thanks to Professor Pauling for his continued helpful interest in the investigation.

* Contribution No. 1711. This work was supported in part by the Office of Naval Research under Contract N6-onr-24423.

1 Bauer, S. H., and Pauling, L., J. Am. Chem. Soc., 58, 2403 (1936).

2 (a) Price, W. C., J. Chem. Phys., 16, 894 (1948): 15, 614 (1947). (b) See also Hedberg, K., and Schomaker, V., J. Am. Chem. Soc., 73, 1482 (1951).

3 Kasper, J. S., Lucht, C. M., and Harker, D., Acta Cryst., 3, 436 (1950).

4 Hedberg, K., Jones, M. E., and Schomaker, V., J. Am. Chem. Soc., 73, 3538 (1951).

- Dulmage, W. J., and Lipscomb, W. N., Ibid., 73, 3539 (1951).

- Pitzer, K. S., Ibid., 67, 1126 (1945).

${ }^{7}$ See Hedberg, K., and Stosick, A. J., Ibid., 74, 954 (1952). 
8 Private communication.

- Pitzer, K. S., and Pimentel, G. C., private communication.

${ }^{10}$ Taylor, W. J., Beckett, C. W., Tung, J. Y., Holden, R. B., and Johnston, H. L., Phys. Rev., 79, 234 (1950).

11 We had at first rejected the tetragonal pyramid (see reference 4 ), mainly because its relative number of $B \cdots B$ interactions and relative $B \cdots B$ distance $(1.74 \sqrt{2}=2.46$ $\AA$.) are in disagreement with this false interpretation of the $2.57 \AA$. peak and because, in view of this interpretation, little attention was given to the (crucial) disposition of the hydrogen atoms. Our initial failure to recognize the $B-B$ split also contributed to the argument on the apparent $B-B-B$ angle (i.e., the ratio 2.57/1.74). Actually, the halfwidth of the $1.74 \AA$. peak is somewhat greater than would be expected from our convergence factor for zero split (although less than for a $0.10 \AA$. split). We overlooked this, failing to consider how little widening would result from distance splits up to $0.10 \AA$., especially in the absence of a well-resolved, sharp term to make the slow decline of the split-term contribution obvious in the appearance of the photographs. (In reference 13 the radial distribution distances were misquoted as $1.76 \AA$. and $2.62 \AA$.; also, in the discussion of the puckered five-membered ring model, it was not made clear that for the theoretical curve mentioned the hydrogen terms were neither based on an actual model nor complete. The complete curve was no longer promising; the original curve, lacking the B-B split, of course never was a good fit to the outer part of the pattern.)

12 Hrostowski, H. J., Myers, R. J., and Pimentel, G. C., J. Chem. Phys., 20, 518 (1952).

${ }^{13}$ Schomaker, V., J. chim. Phys., 46, 262 (1949).

14 Pauling, L., J. Am. Chem. Soc., 69, 542 (1947).

15 Hedberg, K., Ibid., in publication.

${ }^{16}$ Private communication; see references 4 and 5.

${ }_{17}$ Clark, H. K., and Hoard, J. L., J. Am. Chem. Soc., 65, 2115 (1943); Zhdanov, G. S., and Sevast'yanov, N. G., Compt. rend. acad. sci. U. S. S. R., 32, 432 (1941).

${ }^{18}$ Hoard, J. L., Geller, S., and Hughes, R. E., J. Am. Chem. Soc., 73, 1892 (1951).

${ }^{19}$ Our values for $\mathrm{B}_{6} \mathrm{H}_{9}$ are $\angle \mathrm{B}_{1} \mathrm{~B}_{2} \mathrm{~B}_{2}=58^{\circ}, \angle \mathrm{B}_{2} \mathrm{~B}_{2} \mathrm{H}_{3}=48^{1} / 2^{\circ}$, and $\angle \mathrm{H}_{3} \mathrm{~B}_{2} \mathrm{H}_{3}=$ $113^{\circ} ; \angle \mathrm{B}_{1} \mathrm{~B}_{2} \mathrm{H}_{3}=106^{\circ}, \angle \mathrm{B}_{2} \mathrm{~B}_{2} \mathrm{H}_{3}=113^{\circ}$, and $\mathrm{B}_{2} \mathrm{~B}_{2} \mathrm{~B}_{2}=90^{\circ}$; and $\angle \mathrm{H}_{2} \mathrm{~B}_{2} \mathrm{~B}_{1}=120^{\circ}$, $\angle \mathrm{H}_{2} \mathrm{~B}_{2} \mathrm{~B}_{2}=134^{\circ}$, and $\angle \mathrm{H}_{2} \mathrm{~B}_{2} \mathrm{H}_{3}=108^{\circ}$. Here the four-membered ring, the differences of $B-H$ (bridge) and $B-B$ bond lengths, and the evident need for large $\angle \mathrm{H}_{3} \mathrm{~B}_{2} \mathrm{H}_{8}$ to provide a reasonably long $\mathrm{H}_{3} \cdots \mathrm{H}_{3}$ distance, which we presume to be non-bonding, are obviously serious constraints; nevertheless, the average external angle is $121^{\circ}$, in surprising agreement with the ideal value. Also, our value $120^{\circ}$ for $\angle \mathrm{B}_{1} \mathrm{~B}_{2} \mathrm{H}_{2}$ approximately equalizes the external angles, and the smaller, crystal value of $115^{\circ}$, which by the quoted $\pm 5^{\circ}$ limit of error is considerably more reliable than ours, equalizes the apparent strains very well indeed.

${ }^{20}$ Rundle, R. E., J. Am. Chem. Soc., 69, 1327 (1947).

${ }^{21}$ Dunitz, J. D., and Schomaker, V., submitted for publication in the Journal of Chemical Physics. 